\title{
Hollow fibres integrated in a microfluidic cell culture system
}

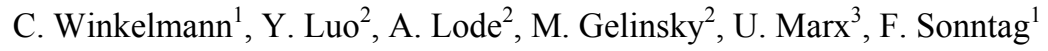 \\ ${ }^{1}$ Fraunhofer Institute for Material and Beam Technology IWS, Dresden, Germany; \\ claudia.winkelmann@iws.fraunhofer.de \\ ${ }^{2}$ Technische Universität Dresden, Centre for Translational Bone, Joint and Soft Tissue Research, Dresden, Germany \\ ${ }^{3}$ Technical University Berlin, Institute of Biotechnology, Berlin, Germany
}

\begin{abstract}
For in vitro drug screening a modified perfusion micro-bioreactor system with integrated hollow fibres will be demonstrated. This biocompatible system consists of an integrated closed flow circuit that includes reservoirs and pneumatic micro pumps. Additional optical online-monitoring devices allow the observation during the cell cultivation. The embedded hollow fibre system which acts as cell carrier consists of a biodegradable biopolymer. One option to fabricate such 3D structures is the technology of Organ Printing which is realised by an adapted rapid prototyping system entitled 3D Scaffold Printer. With this device specimens consisting of tubes with a diameter smaller than 2 mm can be prepared. It should be possible to cultivate the hollow fibres inside and outside with different kinds of cells and therefore generate models of complex tissues.
\end{abstract}

\section{Introduction}

Organ typical tissue cultures have been already established in limited fields of regenerative medicine. Artificial skin tissues, cartilage and bone implants could be developed successfully in the last years. The development of such complex three-dimensional tissue cultures is becoming also more and more important for the pharmaceutical and biochemical industry as a substitute for animal experiments. Such an in vitro substance testing requires the assimilative interaction of different cells or tissues within a common cycle in analogy of the human body [1]. Therefore it was our issue to develop a biomimetic 3D tissue culture with an artificial but functional blood vessel system. It should generate a biocompatible and complex three-dimensional microenvironment that supports the growth of tissue cells. A possibility to realise a biofunctional vascular system is the integration of several hollow fibres into the cell carrier [2]. They have to be integrated and connected to a dynamic micro-bioreactor system to ensure the supply of the artificial tissue with oxygen and nutrient. The main challenge of this study was the connection between the biological and technological system [1].

\section{Methods}

\subsection{Perfusion micro-bioreactor system}

The bioreactor system is based on the established MultiOrgan-Chip platform, developed in cooperation between Fraunhofer IWS and the Institute of Biotechnology of Technical University of Berlin. This platform is shown in Image 1 .

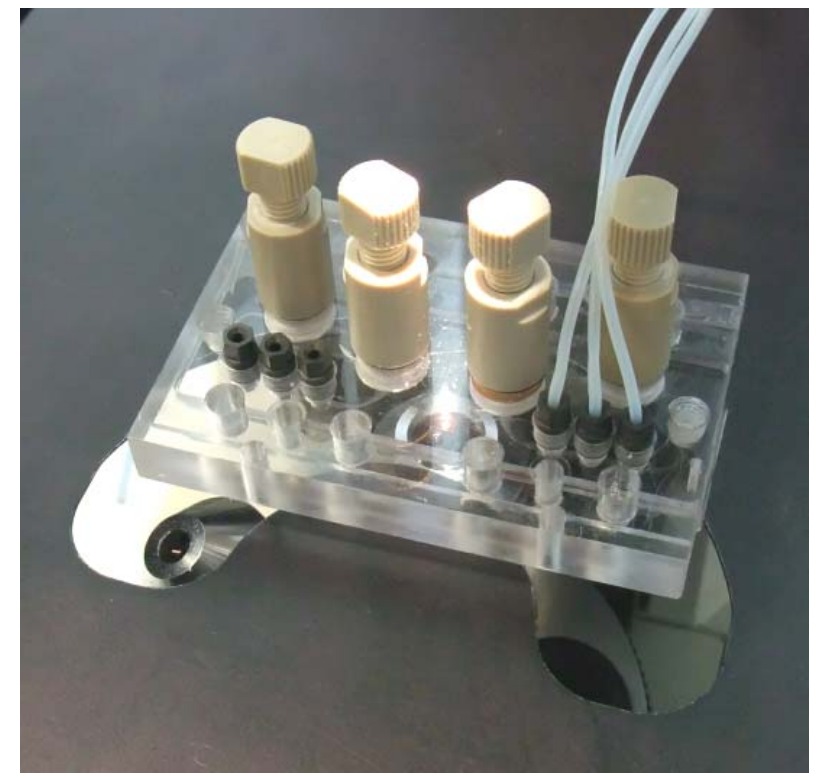

Image 1 Multi-Organ-Chip on microscope

The flow is pneumatically realised and is regulated by an automatic control module. The fluidic parameters can be adjusted by the software system.

With this system it is possible to cultivate different kinds of tissues simultaneously due to the integration of different cell culture chambers, fluidic reservoirs, valve inlets and micro-actors like an automatic pneumatic pump system (Image 2). Over a closed flow circuit that rebuilds a microenvironment the cells can interact with each other [1]. Currently, it includes several replaceable cell inserts for cultivation. But due to large diffusions paths it is difficult to ensure the supply of oxygen and nutrient despite the utilisation of a perfusion system.

A solution for the limitation might be the integration of a hollow fibres system. 

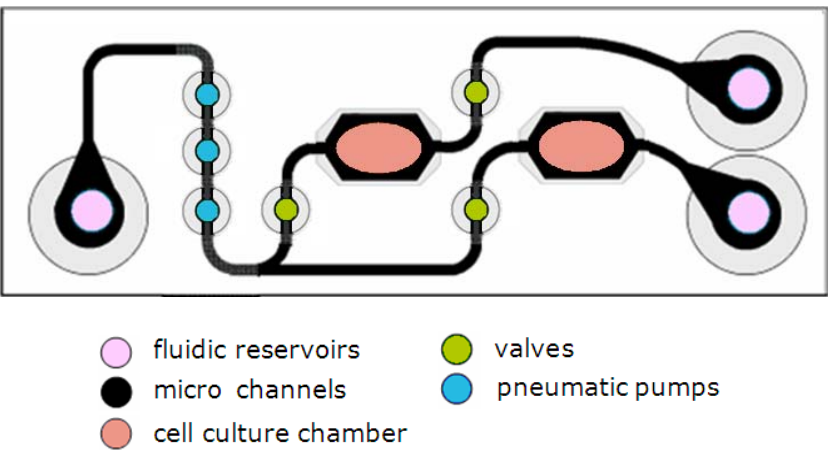

Image 2 Schematic representation of the layout of a self contained Multi-Organ-Chip

\subsection{D Scaffold Printer}

The 3D Scaffold Printer (Image 3) is an innovative tool for creation of open porous cell carriers and scaffolds. The device consists of a 3 channel dispensing module, flexible PC software and a high-precision XYZ robotic with a travel range in A3 size. It is possible to structure media variety of biological (biopolymer) or synthetic pasty materials into thin strands [3].

Repeating this strands and arranging them during the process allows the creation of individual porous systems as cell carrier for the three-dimensional cell cultivation. Up to three different components can be dispensed. An accurate XY offset compensation is given by individual lowering of each cartridge. The exact trajectory of each cartridge is monitored by a sensor system.

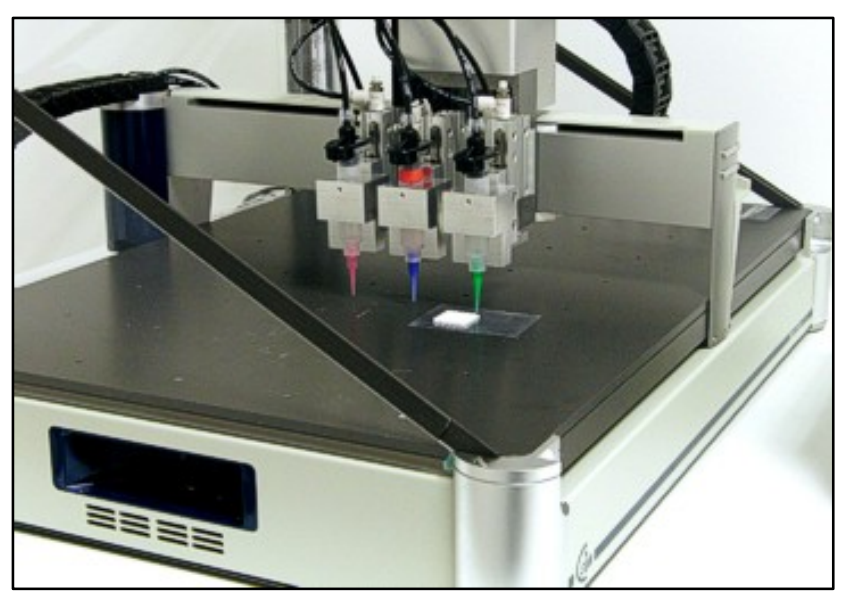

Image 3 3D Scaffold Printer

The 3D Scaffold Printer was developed by the company GeSiM mbH (Großerkmannsdorf) and the dosing module is an in-house development by Fraunhofer IWS.

\subsection{Material}

In cooperation with the Centre for Translational Bone, Joint and Soft Tissue Research of TU Dresden an application-specific cell-matrix-composite based on alginate hydrogels was developed. To cross-link the material an aqueous solution of calcium chloride was applied. Pre- liminary tests have shown the suitability of this material combination. Using pre-sterilised materials and working under sterile conditions allows the simultaneous embedding of living cells during scaffold production.

\section{$3 \quad$ Results}

\subsection{Adjusted perfusion micro-bioreactor system}

For integration of the hollow fibres into the micro perfusion bioreactor the cell culture system had to be adjusted to the new requirements.

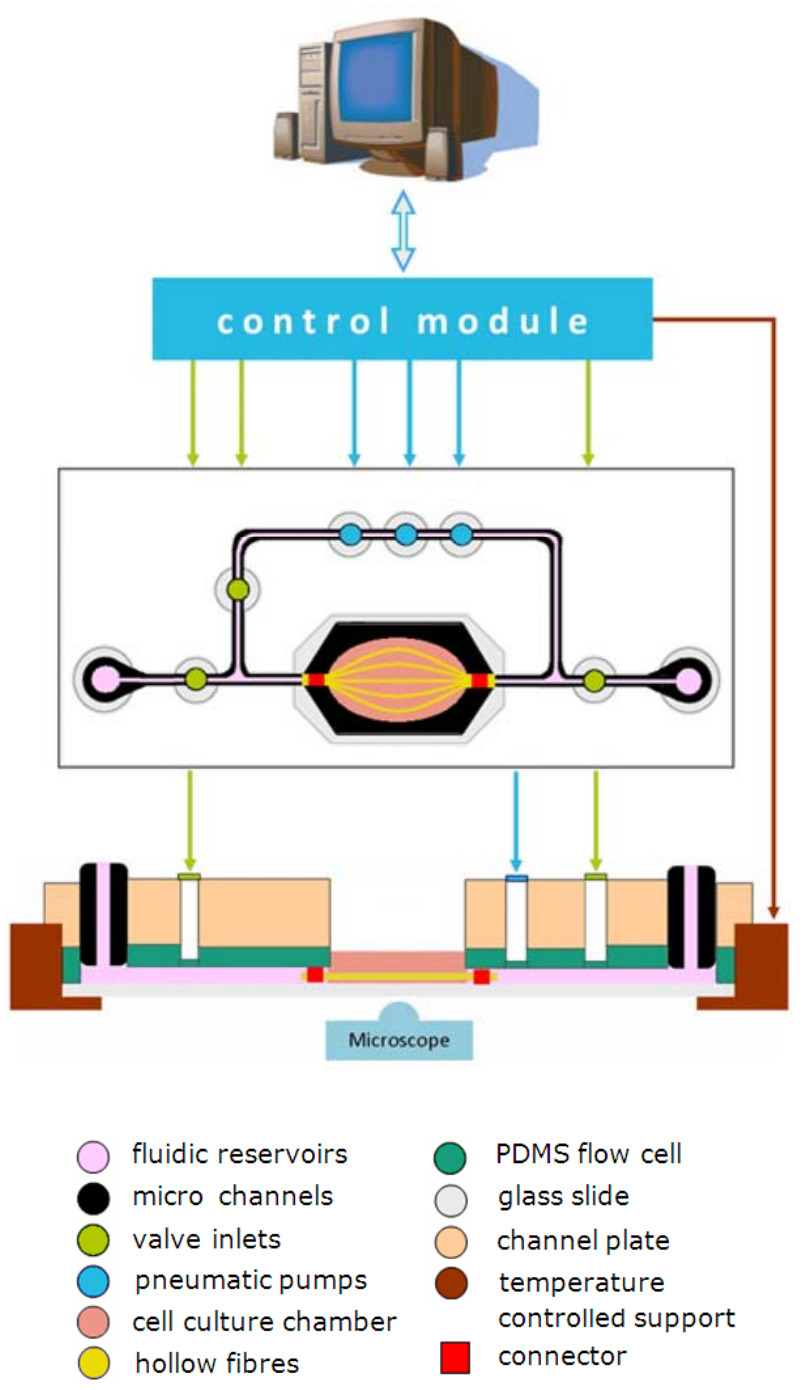

Image 4 Schematic representation of the adjusted fluidic system

For first experiments the fluidic system has been equipped with only one cell culture chamber (Image 4). Its structure was modified to include the hollow fibres. Additionally, the design of the fluidic channels must be specifically engineered to enable the circulation of cell culture media through the whole system. 
The first version of the adjusted perfusion micro-bioreactor system was manufactured successfully (Image 5).

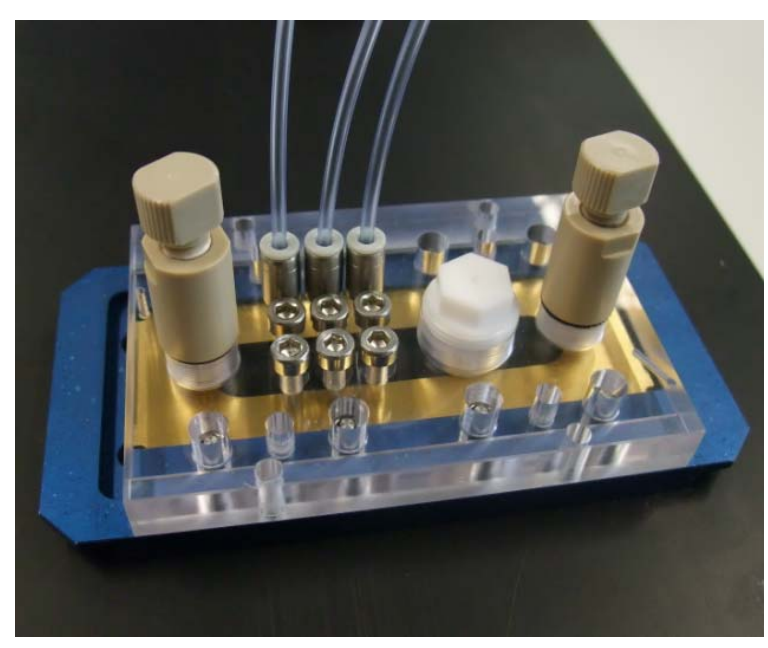

Image 5 Adjusted perfusion micro-bioreactor system

\subsection{Adjusting of the 3D Scaffold Printer dosing module}

By using the 3D Scaffold Printer technology it should also be possible to create hollow fibres. For this it was necessary to adjust the dosing module to the new requirements. (Image 6)

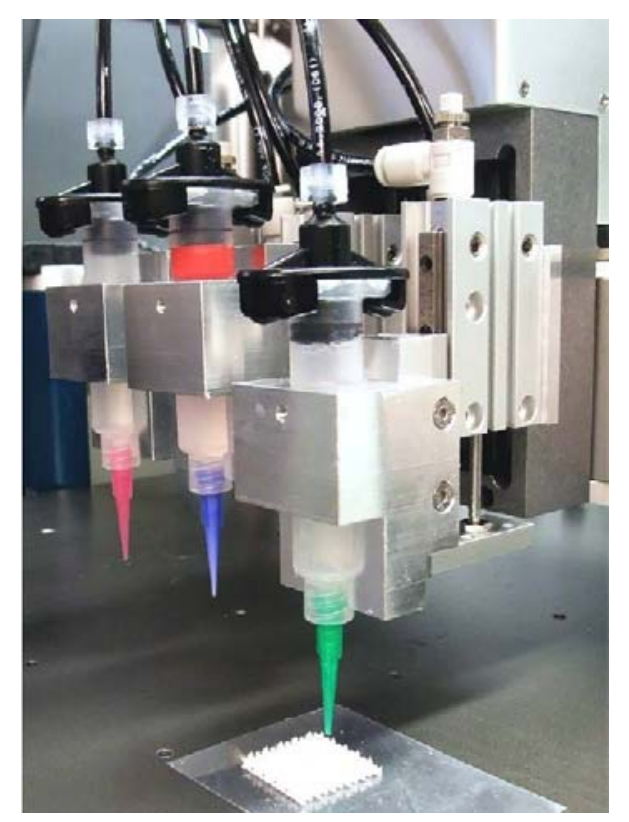

Image 6 Dosing module of the 3D Scaffold Printer

Therefore two cartridges were connected with each other over an engineered dispensing needle system to enable a dual extrusion. One of the needles is fixed inside the other and acts as spacer and has to be exactly aligned for the creation of hollow fibres. Therefore both needles defined the inside and outside diameter of the structure according to the printing materials.

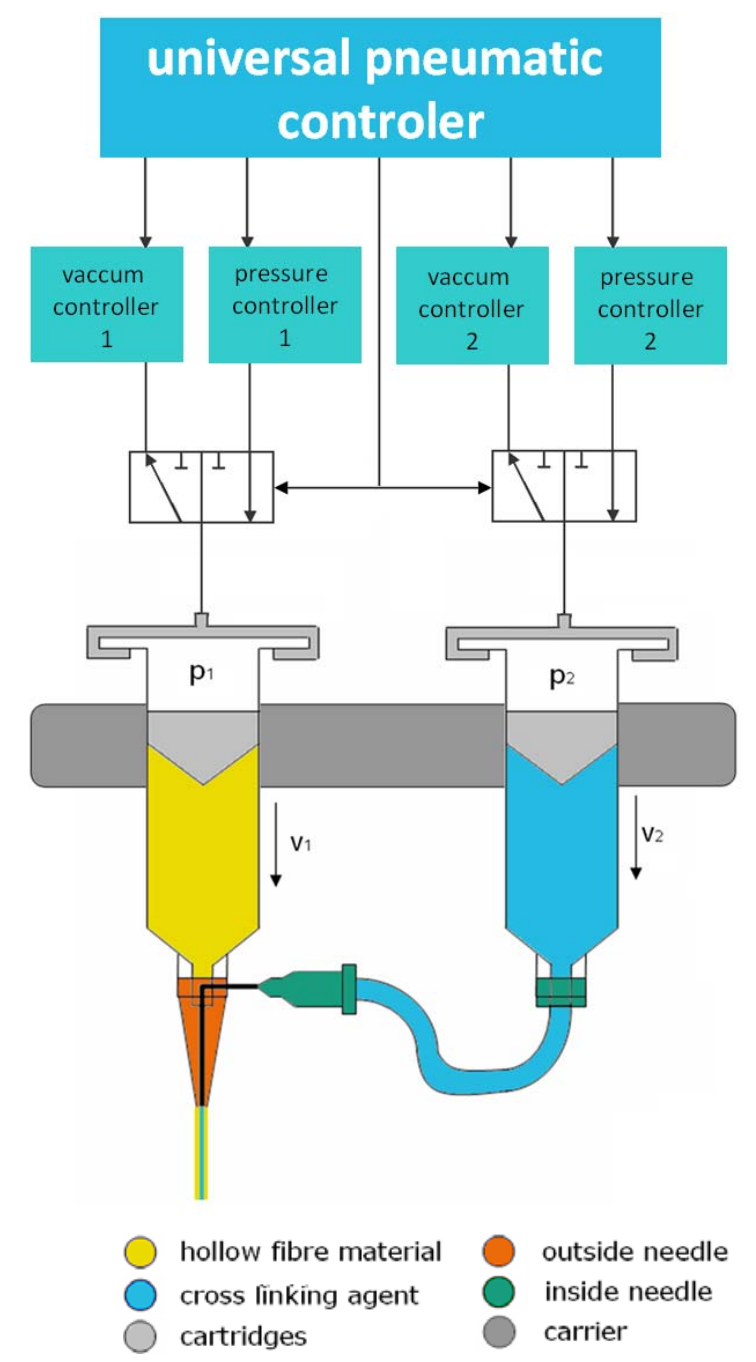

Image 7 Schematic representation of the adjusted dosing module

In a printing process (Image 7) the first cartridge is filled with cell carrier material (e. g. a biopolymer paste) which is extruded by applying a defined pressure. The other cartridge is containing a cross linking agent to cure the cell carrier medium. Since both printing materials usually have different viscosities and therefore different rheological properties, the dosing module was redesigned to generate different pressures in one printing process. Moreover, by using media with a low viscosity it is necessary to attach a negative pressure on the cartridges to prevent uncontrolled leakage of the media. The regulation of switching between pressure and vacuum has to be arranged by the control module. Additionally, both cartridges are controlled in parallel to the dosing process and the trajectory over this module to ensure a continuous structure production. 


\subsection{Plotted hollow fibres}

In response to the adjustment of the dosing module the first hollow fibres based on the alginate cell-matrixcomposite could be produced successfully by using the $3 \mathrm{D}$ Scaffold Printer technology (Image 8 and 9).

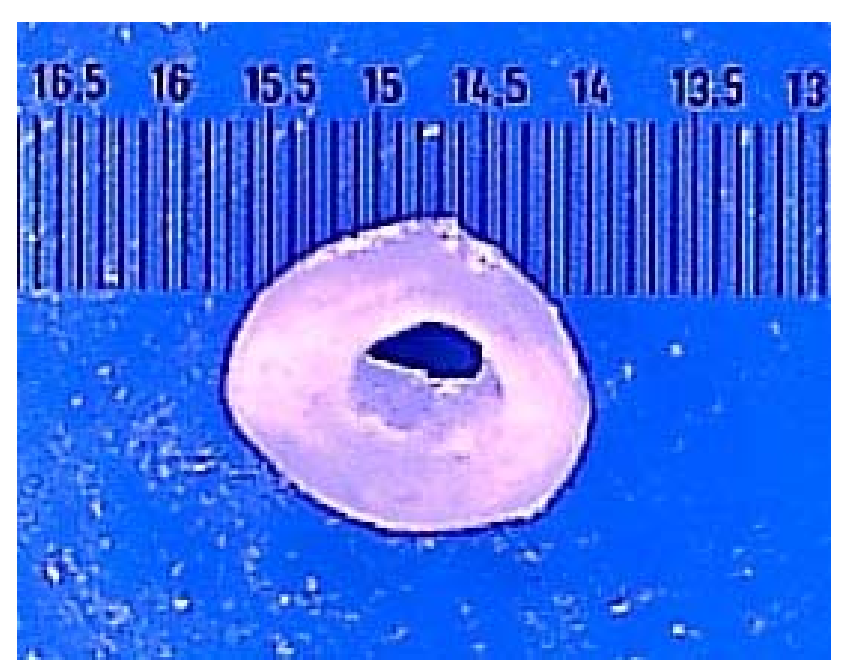

Image 8 Cross section of a plotted hollow fibre

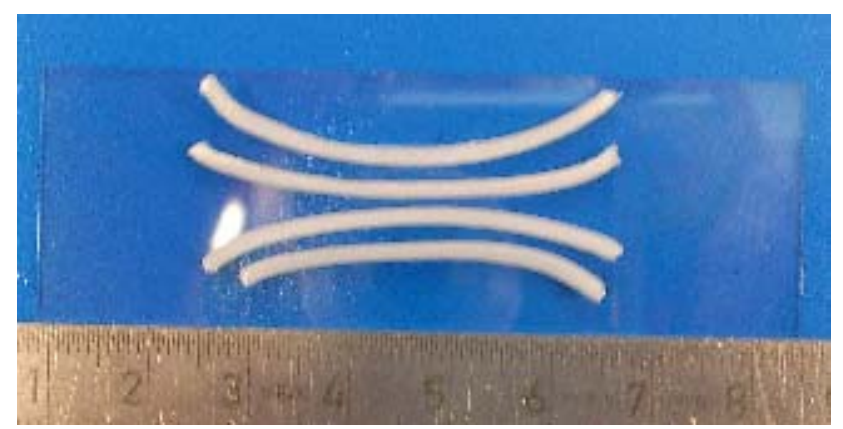

Image 9 Top view of plotted hollow fibres

First produced hollow fibres could be realised with a minimal outside diameter of ca. $720 \mu \mathrm{m}$ and an inside diameter of ca. $250 \mu \mathrm{m}$ by using a dosing needle with an inside diameter of $840 \mu \mathrm{m}$ and an integrated thorn with an outside diameter of $520 \mu \mathrm{m}$.

The produced hollow fibres could also be successfully integrated into the newly developed bioreactor system.

\section{Conclusion}

With the novel technology platform a new powerful tool for research and development in the area of tissue engineering, drug screening and cytotoxicity tests will be available.

The next item is the assessment of the suitability and steadiness of the hollow fibres during perfused cell culture conditions. Moreover, studies will be continued related to flow behaviour and diffusion properties as a function of pressure applied to the fluidic system. To expand the applicability of the system the results shall also be transferred to other biopolymers.

\section{Acknowledgements}

The authors gratefully acknowledge the financial support by the European Union, the Federal State of Saxony and the ESF (European Social Fond).

\section{References}

[1] Sonntag, F., Gruchow, M., Wagner, I., Lindner, G., Marx, U.: Miniaturisierte humane organtypische Zellund Gewebekulturen. BIOspektrum 2011

[2] Meyer, W., Engelhardt, S., Novosel, E., Elling, B., Wegener, M., Krüger, H.: Soft Polymers for Building up Small and Smallest Blood Supplying Systems by Stereolithography. J. Funct. Biomater. 2012, 3, 257 268

[3] A. Lode, K. Meißner, Y. Luo, F. Sonntag, S. Glorius, B. Nies, C. Vater, F. Despang, Th. Hanke, M. Gelinsky: Fabrication of porous scaffolds with defined inner and outer morphology by 3D plotting of a pasty calcium phosphate bone cement. J. Tissue Eng. Reg. Med. 2012 (accepted) 